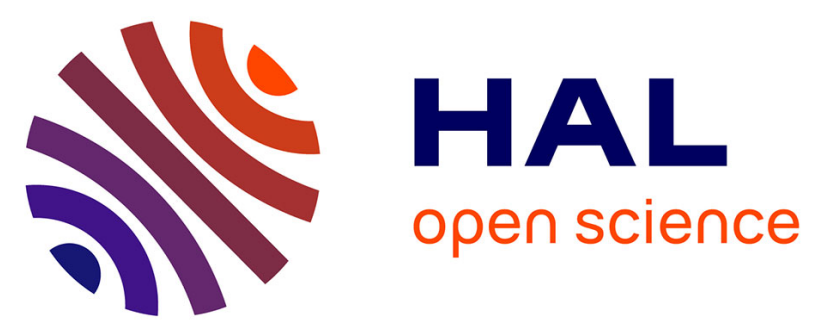

\title{
Epitaxial two-layer graphene under pressure: Diamene stiffer than Diamond
}

Filippo Cellini, Francesco Lavini, Tengfei Cao, Walt A de Heer, Claire Berger, Angelo Bongiorno, Elisa Riedo

\section{- To cite this version:}

Filippo Cellini, Francesco Lavini, Tengfei Cao, Walt A de Heer, Claire Berger, et al.. Epitaxial two-layer graphene under pressure: Diamene stiffer than Diamond. FlatChem, 2018, 10, pp.8-13. 10.1016/j.flatc.2018.08.001 . hal-02144581

\section{HAL Id: hal-02144581 \\ https://hal.science/hal-02144581}

Submitted on 30 May 2019

HAL is a multi-disciplinary open access archive for the deposit and dissemination of scientific research documents, whether they are published or not. The documents may come from teaching and research institutions in France or abroad, or from public or private research centers.
L'archive ouverte pluridisciplinaire HAL, est destinée au dépôt et à la diffusion de documents scientifiques de niveau recherche, publiés ou non, émanant des établissements d'enseignement et de recherche français ou étrangers, des laboratoires publics ou privés. 


\title{
Epitaxial two-layer graphene under pressure: Diamene stiffer than Diamond
}

Filippo Cellini ${ }^{1,2 \S}$, Francesco Lavini ${ }^{1,2,3 \S}$, Tengfei $\mathrm{Cao}^{1,6}$, Walt de Heer ${ }^{4,7}$, Claire Berger ${ }^{4}$, Angelo Bongiorno $^{1,3,6}$ and Elisa Riedo ${ }^{1,2,3,5^{*}}$

\author{
${ }^{1}$ Advanced Science Research Center, City University of New York, New York 10031, NY, USA. \\ ${ }^{2}$ Tandon School of Engineering, NYU, Brooklyn 11201, NY, USA. \\ ${ }^{3}$ CUNY Graduate Center, Ph.D. Program in Physics and Chemistry, New York 10016, NY, USA. \\ ${ }^{4}$ School of Physics, Georgia Institute of Technology, Atlanta, GA, USA \\ ${ }^{5}$ Physics Department, City College of New York, City University of New York, New York 10031, NY, USA. \\ ${ }^{6}$ Department of Chemistry, College of Staten Island, City University of New York, Staten Island 10314, NY, USA. \\ ${ }^{7}$ TICNN, Tianjin University, Tianjin, China \\ $\S$ These Authors contribute equally to this work. \\ ${ }^{*}$ Corresponding author: elisa.riedo@nyu.edu
}

The unprecedented ultrahigh interlayer stiffness of supported two-layer epitaxial graphene on silicon carbide $(\mathrm{SiC})$ has been recently reported by our research group. We found that under localized pressure a two-layer epitaxial graphene behaves as an ultra-hard and ultrastiff coating, showing exceptional mechanical properties that far exceed those of bare SiC. Density functional theory (DFT) calculations indicate that this unique behavior stems from a $s p^{2}$-to-s $s p^{3}$ reversible phase transition of carbon films under compression, leading to a single-layer diamond-like structure that we called diamene. In this paper, force versus indentation depth curves from high-resolution nanoindentation experiments of CVD diamond and sapphire are carried out and compared to those obtained from two-layer epitaxial graphene on SiC. These new measurements confirm that the stiffness of epitaxial graphene is larger than that exhibited by CVD diamond and sapphire substrates. Our measurements show that areas of the film consisting of buffer layer plus one, or at most two, additional graphene layers are the ones most likely to exhibit phasechanging behaviors and larger-than-diamond stiffness.

\section{INTRODUCTION}

The outstanding mechanical properties of graphene have been investigated for more than a decade. Probably the most relevant experimental investigation to date of the nanomechanical properties of graphene was reported in 2008 in Science [1]. In this experiment, the elastic modulus and strength of mechanically exfoliated monolayer graphene was measured by nanoindentation in an atomic force microscope (AFM). Experimental results demonstrated the extremely high in plane stiffness of monolayer graphene, of the order of $1 \mathrm{TPa}$, and the outstanding membrane strength of above $100 \mathrm{GPa}$. These results opened avenues to further investigation of the mechanical properties of exfoliated graphene [2-4] and graphene oxide [5], whereby different studies attempted to dissect the structural characteristics of graphene flakes that are responsible for their incredible mechanical response.

Despite being the most reliable and cost-effective technique for producing high quality crystals, mechanical exfoliation of graphene from graphite produces only sparse isolated flakes, with no precise control on dimensions and thickness distribution. This limitation has hampered application of mechanically exfoliated graphene to large-scale devices and technological platforms. For this reason, epitaxial growth of graphene on hexagonal substrates has gathered the increasing attention 
of the scientific community as a viable alternative to mechanical exfoliation [6]. Several production methods have been explored, some of them involving the growth of epitaxial graphene (EG) on transition metal surfaces via chemical vapor deposition (CVD) [7], other providing a surface-confined growth through precise control of temperature ramping (temperature programmed growth, or TPG [8]), or exploiting the carbon solubility on suitable substrates [9].

In this regards, the preparation of epitaxial graphene by thermal decomposition of silicon carbide substrate $(\mathrm{SiC})$ is considered as a solid route to the controlled synthesis of large scale graphene films for several technological applications [10]. Recent developments in this technique have led to an accurate control over dimensions and thicknesses of the films, making possible to obtain continuous and homogenous single layer graphene domains up to several tens of micrometers in length and width [11].

Fundamental characteristic of this growth process is the formation of an interfacial layer between the graphene layers and the $\mathrm{SiC}$ substrate, which is called buffer layer. Because of the strong covalent bonds with the $\mathrm{SiC}$, the buffer layer presents substantially different properties from graphene. In fact, although maintaining a graphite-like honeycomb structure, the buffer layer does not display the $\Pi$ bands of graphene [12], is semiconductive [13], and is highly charged [14]. Layers grown on top of the buffer layer act like few-layer graphene structures, stacking on top of the buffer and presenting the same electronic structure of graphene. Therefore, the presence of the buffer layer is known to play a fundamental role in shaping the properties of few layer epitaxial graphene on $\mathrm{SiC}$.

Very recently, AFM experiments and density functional theory (DFT) calculations explored the mechanical properties of few-layers epitaxial graphene film grown on $\mathrm{SiC}(0001)$, particularly focusing on the interlayer response of the material to nanoindentation $[15,16]$. By harnessing the sub-ångström-resolution of modulated nano-indentation (MoNI), these experiments demonstrated that at room temperature and under pressure, two-layer (2-L) epitaxial graphene films display stiffness much larger than the $\mathrm{SiC}$ substrate. Here, two-layer epitaxial graphene refers to a single layer of graphene grown on top of the interfacial buffer layer. The ultra-high stiffness of these films was explained through a detailed set of DFT calculations showing that 2-L epitaxial graphene under pressure would likely undergo a homogenous phase transition from a $s p^{2}$-hybridized graphitic to a $s p^{3}$ - diamond-like structure. These results strongly supported our hypothesis that the ultrahigh stiffness observed in the experiments was indeed associated with the formation of a new material at the interface between $\mathrm{SiC}$ and graphene, here-after called diamene.

Our previous study demonstrated for the first time the ultra-high stiffness of diamene. However, in our report, we did not provide an estimation of the effective stiffness of this new diamond-like phase by direct comparison with other stiff materials. In this work, we further explore the properties of diamene, comparing its stiffness with that of two hard substrates, namely CVD diamond and sapphire. These new experimental observations confirm that the stiffness of diamene is indeed comparable or even higher than the stiffness of bulk diamond and is of the order of 1 $\mathrm{TPa}$.

The rest of the paper is organized as follows. In Section 2, we discuss the growth process of epitaxial graphene and the procedure for MoNI ( $\AA$-indentation). Furthermore, we analyze the challenges related to the identifications of ultrahigh stiffness regions in few-layer epitaxial graphene samples. In Section 3, we report the topography and frictional properties of an epitaxial graphene sample where the possible formation of diamene was observed. The comparison between 
indentation curves of diamene, buffer layer, and two other reference materials, CVD diamond and sapphire is also given. In Section 4, we present our conclusions.

\section{MATERIALS and METHODS}

\subsection{Growth and characterization of epitaxial graphene films on $\mathrm{SiC}$}

Epitaxial graphene samples employed in these experiments are grown on on-axis $4 \mathrm{H}-\mathrm{SiC}$ wafer by the confinement-controlled sublimation method [17-19]. In its fundamental incarnation, the confined sublimation method is composed of two phases: sample preparation and graphene growth in a high temperature furnace. In the first phase, $\mathrm{SiC}$ wafers are initially cut to shape (approximately $5 \times 5 \mathrm{~mm}^{2}$ samples are employed in this work) and polished on the growth face. After that, samples are placed in the furnace under Argon atmosphere for graphene growth. Temperature and time are optimized to produce multilayer graphene layers on the $\mathrm{SiC}(000-1)$ face (C-face) and a single graphene layer on the $\mathrm{SiC}(0001)$ face (Si-face) [20]. This specific growth process is realized in two steps, a first step around $1450^{\circ} \mathrm{C}$ for 7 minutes and a second step at 1550 ${ }^{\circ} \mathrm{C}$ for 20 minutes.

A TEM micrograph of few graphene layers grown on the Si-face is displayed in Figure 1(a). A schematic and molecular model of the epitaxial graphene structure is displayed in Figure 1(b). Atomically thin epitaxial graphene films up to 5 atomic layers, and buffer layer, are generally grown on the $\mathrm{SiC}(0001)$ face ( $\mathrm{Si}$-face) of the $\mathrm{SiC}$, while films with more than ten layers are grown on the carbon face of $\mathrm{SiC}(000-1)$. The first grown carbon layer on $\mathrm{Si}$-face is known as the buffer layer. The buffer layer is characterized by a graphene-like honeycomb atomic structure with about $30 \%$ of its carbon atoms bonding to the $\mathrm{Si}$ atoms of the $\mathrm{SiC}(0001)$ face. This particular structure gives the buffer layer a unique set of properties, such as semiconducting electronic properties due to its interaction with $\mathrm{SiC}(0001)$ surface.

\subsection{Stiffness measurements by Å-indentation MoNI}

MoNI is an Atomic Force Microscopy (AFM)-based force spectroscopy technique which allows for sub-ångström resolution indentation measurements. We specifically select MoNI for our experiments to overcome limitations in the study of atomically thin two-dimensional (2D) materials that are introduced by other nanoindentation methods [21, 22]. Traditional nanoindentation measurements usually employ indentation depths of the order of few hundred nanometers, whereby small residual deformations are likely to be produced on the surface of the test sample. In the case of few layer graphene films in Figure 1(a) and (b), these indentation depths substantially exceed the thickness of the film, compromising the accuracy and reliability of the measured stiffness, which is not characteristic of the thin film, but arises from the predominant contribution of the substrate.

Different from traditional techniques, MoNI employs extremely small (sub-ångström) vertical oscillations to probe the surface of the sample (see Figure 2(a)). The oscillatory displacement $\left(\Delta z_{\text {piezo }}\right)$ is imposed to the cantilever-tip system by using a small sinusoidal voltage $(<0.4 \mathrm{mv})$ applied to the piezotube of the AFM (Agilent PicoPlus AFM) by a lock-in amplifier (Stanford Research Systems, SR830), while the contact force $\left(F_{z}\right)$ between tip and sample is maintained constant by the feedback loop of the AFM. In further details, the tip is first brought in contact with the surface. Once the contact force has reached $F_{z} \sim 10^{2} \mathrm{nN}$, the tip is slowly retracted $(0.01 \mathrm{~nm} / \mathrm{s})$ from the surface while oscillating with amplitude $\Delta z_{\text {piezo }}(<1 \AA)$. The variation in the normal force 
$\left(\Delta F\left(F_{z}\right)\right)$ induced by the imposed oscillation is measured while reducing $F_{z}$, until the tip loses contact with the surface.

The stiffness of the contact $k_{\mathrm{cont}}(\mathrm{Fz})=\left(\Delta z_{\text {piezo }} / \Delta F\left(F_{z}\right)-1 / k_{\mathrm{lev}}\right)^{-1}$ is recorded at different decreasing loads ( $k_{\text {lev }}$ being the spring constant of the AFM cantilever), allowing high-resolution force $\left(F_{z}\right)$ indentation $\left(z_{\text {indent }}\right)$ curves to be constructed by computing the following integral:

$$
z_{\text {indent }}=z_{\mathrm{P} 0}+\int_{F_{P 0}}^{F_{z}} \frac{d F_{Z}^{\prime}}{k_{\operatorname{cont}}\left(F_{z}^{\prime}\right)}
$$

where $F_{P 0}$ is the pull-out force measured by the AFM when the tip loses contact with the sample's surface at $z_{\mathrm{P} 0}$.

The stiffness of the contact between the tip and the sample can be identified by fitting the indentation curve with the classical Hertz model:

$$
F_{z}=\frac{4 E^{*} \sqrt{R}}{3} z_{\text {indent }}^{3 / 2}
$$

where $R$ is the tip radius and $E^{*}=\left(\left(1-v^{2} \text { tip }\right) / E_{\text {tip }}+\left(1-v^{2}\right) / E\right)^{-1}$ is the effective Young modulus of the tip/sample contact, dependent on the Poisson's ratios and elastic modulus of both tip ( $\left.v_{\text {tip }}, E_{\text {tip }}\right)$ and sample $(v, E)$. Despite its simplicity, the Hertz equation turns out to be accurate in matching the experimental data [1].

Indentation curves measured on 2-L and 10-layer epitaxial graphene are reported in Figure 2(b), together with the indentation curve obtained for a bare $\mathrm{SiC}$ substrate employed for graphene growth. According to the Hertz formula in equation (2), when tip properties ( $\left.v_{\text {tip }}, E_{\text {tip }}\right)$ and shape (contact radius, $R$ ) are maintained constant across different experiments, the slope of the indentation curves is directly proportional to the stiffness of the surface, with a steeper indentation curve indicating a stiffer surface. Therefore, the substantially steeper slope of the 2-L graphene curve indicates that this film is much stiffer than SiC. As discussed in our former work [16], we associate this surprising result to the non-permanent transformation of 2-L graphene into an ultrastiff diamene layer due to the pressure of the AFM tip.

In [15], direct quantification of multi-layer (10-layer) graphene stiffness (E 33 GPa) was successfully performed using equation (2), by comparing the stiffness of the film to the stiffness of the bare $\mathrm{SiC}$ substrate. However, a similar identification performed on 2-L graphene indentation curves in Figure 2(b) would give a stiffness of diamene substantially higher than that of diamond [16], which is one of the stiffest material known to mankind. For this reason, we report herein diamene indentation curves measured in parallel to indentation curves of other ultrastiff materials, namely CVD diamond and a sapphire crystal. Through direct comparison, accurate estimation of diamene indentation modulus can be performed.

In what follows, sub-ångström resolved nanoindentation curves of 2-L graphene are obtained through MoNI. In these experiments, a diamond-coated Si tip (Nanosensor DT-NHCR) is brought into hard contact with the surface of the sample, reaching normal loads up to $300 \mathrm{nN}$, corresponding to pressure of around $10 \mathrm{GPa}$, considering the contact area between the spherical tip and the flat surface (see Figure 2(a)). We use the MoNI technique at room temperature to probe the transverse mechanical response of 2-L epitaxial graphene and compare it with those of bulk (0001) sapphire $\left(\mathrm{Al}_{2} \mathrm{O}_{3}\right)$ and a thin film of CVD (111) diamond $(2.2 \mu \mathrm{m}$ on $\mathrm{Si}$ substrate). The indentation is 
performed in 5-6 different positions on each sample, to have an estimation of the uncertainty of the data acquired.

\subsection{Identification of graphene/diamene structures and their mechanical response}

Although thermally induced growth of the epitaxial graphene on SiC substrate has been widely investigated $[10,11]$, the perfect control of thickness distribution of graphene structure is still very challenging, involving complex dynamics and requiring precise recipes. Therefore, it is difficult to obtain homogeneous and uniformly covered samples of epitaxial graphene, in particular when few layer graphene samples are fabricated. Due to rapid growth rates in the initial stages of the process, large few-layer graphene regions usually coexist with multilayer regions, as well as uncovered buffer layers domains [12].

From a nanomechanical standpoint, these regions have very different properties. As discussed in the previous section, multilayer epitaxial graphene (10 layers or more) presents mechanical stiffness similar to the interlayer stiffness of graphite (approximately $30 \mathrm{GPa}$ ). On the other hand, the buffer layer has an indentation modulus that is almost the same as SiC (approximately 440 GPa) [16], which can be explained with the partial graphitization of the buffer layer and its strong covalent bonding to the underlying $\mathrm{SiC}$ structure. The formation of diamene is observed only in few-layer graphene regions. However, this ultrahigh stiffness is not systematically observed at each point of the sample, which led us believe that only specific graphene structures/number of layers can lead to the formation of ultrastiff diamene.

DFT calculations suggest that the formation of diamene is possible in the case of one graphene layer over the buffer layer and two graphene layers over the buffer layer, but it is highly unlikely when the number of layers exceed four [16]. Indentation data presented in this work are obtained for 2-L epitaxial graphene films. Nevertheless, we are currently working on determining if similar results are found in a three-layer epitaxial graphene film, by developing a methodology to clearly identify in-situ the exact number of layers while performing the MoNI experiments.

In the case of epitaxial graphene, differently from mechanically exfoliated graphene, topographic information is not sufficient to distinguish graphene regions of different thickness. During the growth process, the surface of $\mathrm{SiC}$ substrate undergoes substantial changes, resulting from the sublimation of $\mathrm{Si}$ atoms and consequent rearrangement of $\mathrm{C}$ atoms into a graphitic structure. Additional graphene layers after the first one grow underneath it [13], with the buffer layer being essentially a moving interface between the graphene layers and the SiC substrate. This characteristic of the growth process heavily reflects on the topography, in the sense that the surface height profile does not necessarily follow the effective changes of the graphene thickness.

Friction force microscopy (FFM) can prove to be very useful towards the characterization of the sample structure. It has been demonstrated that friction response varies in epitaxial graphene as a function of thickness and that the friction of the buffer layer far exceeds that of few layers graphene[23]. Mapping the friction response from the surface of the sample can help distinguishing between different graphene domains, opening new avenues for understanding how the number of layers correlates with the formation of diamene and the mechanical properties of the surface.

\section{RESULTS and DISCUSSION}

\subsection{Topography of the EG sample and identification of two-layer graphene regions}


Figure 3 shows a typical atomic force microscopy (AFM) topographic image of the 2-L epitaxial graphene sample studied in this work, together with its corresponding friction force map ${ }^{1}$. Figure 3(a) displays the topography of the epitaxial graphene sample. The structure of the sample displays several vertical terraces resulting from the annealing of $\mathrm{SiC}$, each terrace being separated from the others by clear vertical steps. Graphene growth is known to start at the edges of these steps, and gradually distribute on the $\mathrm{SiC}$ terraces. For this reason, regions close to the step edges usually show lower topography, which is associated to the formation of a multilayer graphene structure (Si atoms sublimate faster near the step edges). Moving from the edges into the terraces, two different regions can be identified: i) regions at higher topography, which we identify as exposed buffer layer in areas where slower sublimation has not allowed the formation of a complete graphene layer; ii) regions at lower topography, which can be identified as the 2-L graphene regions on which we found the ultrastiff diamene.

Figure 3(b) displays the friction force map corresponding to the topographic image in Figure 3(a). In the friction map, it is possible to clearly identify the buffer layer as the high-friction areas being surrounded by the low-friction 2-L epitaxial graphene. Analyzing the values obtained from the two distinct domains, we find that the buffer layer shows a friction force around 9 times larger than the one displayed by 2-L epitaxial graphene. Overall, this ratio agrees with results found in the literature[23]. As expected, higher topographic domains match the bright spots in the friction map, univocally identifying the buffer layer and allowing us to recognize the exposed epitaxial graphene and select suitable areas to perform nanoindentation measurements.

\subsection{Nanoindentation of Diamene, Diamond and Sapphire}

The ultra-high stiffness of 2-L graphene on $\mathrm{SiC}$ has been already established in our previous experiments, but a clear comparison with other ultrastiff materials has not been performed to date. For this purpose, indentation curves are simultaneously acquired on two ultrastiff materials as sapphire (0001) and CVD (111) diamond, and the results are compared with the indentation data of buffer layer and 2-L graphene.

Figure 4(a) displays MoNI indentation curves measured on 2-L epitaxial graphene (diamene) and buffer layer, together with indentation curves of sapphire and CVD diamond. Notably, the slope of the indentation curve of the buffer layer is comparable to the slope of the curve measured on sapphire. On the other hand, a much higher contact force is recorded on regions of the sample with 2-L graphene, leading to a slope of the indentation curve steeper than that of CVD diamond. We associate the ultra-high stiffness of the 2-L graphene regions with the formation of the new ultrastiff diamene phase. We remark that ultra-high stiffness is not observed uniformly everywhere on the sample, and results for mechanical properties can change from region to region. Further investigation is now ongoing to understand which features exactly reveal the presence of diameneforming graphene in different regions of the sample.

Quantitative evaluation of the stiffness of the different materials is performed by fitting the experimental data in Figure 4(a) with Equation (2) using a non-linear regression procedure in Python $^{2}$. Sapphire, taken as the reference material, shows a value of stiffness very close to the expected one [21], with an indentation modulus of $412 \pm 36 \mathrm{GPa}$. For the buffer layer, we compute a stiffness of $498 \pm 26 \mathrm{GPa}$, which is comparable but slightly higher than the expected stiffness of

\footnotetext{
${ }^{1}$ Both topography and friction measurements are performed in contact mode with a Bruker Multimode 8 AFM, using a silicon AFM tip (PPT-CONT Nanosensor) with normal spring constant of $17 \mathrm{~N} / \mathrm{m}$.

${ }^{2}$ For these calculations, we assume for the diamond coated tip $E_{\text {tip }}=1050$ and $v_{\text {tip }}=0.115$.
} 
$\mathrm{SiC}$ (440 GPa). Fitting of the CVD diamond data yields a stiffness of $923 \pm 78 \mathrm{GPa}$. Finally, the stiffness obtained for the diamene film is $1079 \pm 69 \mathrm{GPa}$.

From our analysis, it is clear that diamene structures present a transverse stiffness higher than CVD diamond. This finding agrees with previous micro-hardness experiments [16], where 2-L epitaxial graphene on $\mathrm{SiC}$ proved to be resistant to perforation by a diamond indenter. Upon applying pressure above $17 \mathrm{GPa}$ with a diamond Berkovich indenter, permanent plastic deformation was induced on bare $\mathrm{SiC}$ substrate, while no residual indentation mark was found on the 2-L epitaxial graphene grown on the same substrate. In addition, when applying larger loads on 2-L graphene on $\mathrm{SiC}$, the tip broke.

Density functional theory (DFT) calculations have been carried out to shed light on this surprising experimental observation [16]. In Figure 4(b), we report a schematic of one of possible phase transitions from graphene to diamene, explaining the remarkable hardening effect of the 2-L graphene on $\mathrm{SiC}$. A fundamental result of this analysis is that the $s p^{2}$-to-sp phase transition occurs in 2-L graphene surface under localized pressure, while the rest of the thin film would maintain the graphitic-flexible state. DFT calculations show also that the mechanism depicted in Figure 4(b) is fully reversible and that this transition is possible only for specific number of atomic layers and stacking patterns. Experiments have indeed verified the absence of this stiffening transition in the case of the sole buffer layer, where the stiffness of the graphitic film matches that of $\mathrm{SiC}$ substrate, or in the case of epitaxial graphene with 5 layers or thicker grown on $\mathrm{SiC}$ [2]. The phase transformation seems thus to require a specific topology and stacking of the graphene film as well as a mechanism to saturate a critical fraction of the surface dangling bonds at the interfaces, in particular the one between diamene and substrate. In view of this, the presence of the buckled buffer layer in direct contact with incommensurable and reactive Si-face of the SiC substrate may play a key role in the evolution of the phase transition.

\section{CONCLUSION}

In conclusion, sub-ångström resolution indentation experiments show that, at room temperature, under the pressure of about $10 \mathrm{GPa}$, a 2-L epitaxial graphene film grown on $\mathrm{SiC}(0001)$ behaves as a diamond-stiff coating, exhibiting mechanical stiffness higher than CVD diamond. DFT simulations show that, being independent on stacking patterns, a homogeneous reversible phase transition of 2-L epitaxial graphene is achievable under pressures of experimental indentation. However, in multilayer graphene films, this transformation process is prohibited by stacking constraints [16].

Up to now the microscopic details of the graphene-diamene structural transition remain elusive. But this discovery opens up a series of possibilities in the field of mechanical technologies, including, in particular, applications in ultra-hard and ultra-light coatings and composites. A phasechanging 2D carbon films can have great potential as an entirely new class of ultra-light protective coatings and armors for military and law enforcement personnel, vehicles, and devices. But in order to step into the applicability of such technology, a deeper understanding of the mechanisms behind the phase transition, as well as a broader characterization of the mechanical properties of this novel carbon forms, are absolutely necessary. This can be achieved, for example, by studying different type of graphene films and substrates, including different growth processes, or by exploring other types of mechanical experiments, such as dynamic high-strain rate and ballistic testing. Finally, both experiments and mechanical modeling studies are also necessary to understand whether the same stiffening effect can be exhibited by 2D atomically thin films other 
than graphene.

\section{ACKNOWLEDGMENTS}

We acknowledge the support from the Office of Basic Energy Sciences of the US Department of Energy (grant no. DE-SC0016204).

\section{REFERENCES}

1. Lee, C., et al., Measurement of the elastic properties and intrinsic strength of monolayer graphene. Science, 2008. 321(5887): p. 385-388.

2. Scarpa, F., S. Adhikari, and A. Srikantha Phani, Effective elastic mechanical properties of single layer graphene sheets. Nanotechnology, 2009. 20(6): p. 065709.

3. Zhang, B., L. Mei, and H.F. Xiao, Nanofracture in graphene under complex mechanical stresses. Applied Physics Letters, 2012. 101(12).

4. Zandiatashbar, A., et al., Effect of defects on the intrinsic strength and stiffness of graphene. Nat Commun, 2014. 5: p. 3186.

5. Suk, J.W., et al., Mechanical Properties of Monolayer Graphene Oxide. ACS Nano, 2010. 4(11): p. 6557-6564.

6. Tetlow, H., et al., Growth of epitaxial graphene: theory and experiment. Physics reports, 2014. 542(3): p. 195-295.

7. Ago, H., et al., Epitaxial chemical vapor deposition growth of single-layer graphene over cobalt film crystallized on sapphire. Acs Nano, 2010. 4(12): p. 7407-7414.

8. Coraux, J., et al., Growth of graphene on Ir (111). New Journal of Physics, 2009. 11(2): p. 023006.

9. Liu, X., et al., Segregation growth of graphene on $\mathrm{Cu}-\mathrm{Ni}$ alloy for precise layer control. The Journal of Physical Chemistry C, 2011. 115(24): p. 11976-11982.

10. Berger, C., et al., Ultrathin epitaxial graphite: $2 D$ electron gas properties and a route toward graphene-based nanoelectronics. The Journal of Physical Chemistry B, 2004. 108(52): p. 19912-19916.

11. Emtsev, K.V., et al., Towards wafer-size graphene layers by atmospheric pressure graphitization of silicon carbide. Nature Materials, 2009. 8(3): p. 203-207.

12. Riedl, C., C. Coletti, and U. Starke, Structural and electronic properties of epitaxial graphene on SiC ( $\left.\begin{array}{llll}0 & 0 & 0 & 1\end{array}\right)$ : a review of growth, characterization, transfer doping and hydrogen intercalation. Journal of Physics D: Applied Physics, 2010. 43(37): p. 374009.

13. N. Nair, M., et al., Band gap opening induced by the structural periodicity in epitaxial graphene buffer layer. Nano letters, 2017. 17(4): p. 2681-2689.

14. De Heer, W.A., et al., Epitaxial graphene. Solid State Communications, 2007. 143(1-2): p. $92-100$.

15. Gao, Y., et al., Elastic coupling between layers in two-dimensional materials. Nature Materials, 2015. 14(7): p. 714-720.

16. Gao, Y., et al., Ultrahard carbon film from epitaxial two-layer graphene. Nature Nanotechnology, 2018. 13(2): p. 133-138.

17. de Heer, W.A., et al., Large area and structured epitaxial graphene produced by confinement controlled sublimation of silicon carbide. Proceedings of the National Academy of Sciences of the United States of America, 2011. 108(41): p. 16900-16905.

18. Berger, C., et al., Electronic confinement and coherence in patterned epitaxial graphene. Science, 2006. 312(5777): p. 1191-1196. 
19. Kelly, B.T., Physics of graphite. 1981.

20. Berger, C., et al., Electronic confinement and coherence in patterned epitaxial graphene. Science, 2006. 312(5777): p. 1191-1196.

21. Oliver, W.C. and G.M. Pharr, An Improved Technique for Determining Hardness and Elastic-Modulus Using Load and Displacement Sensing Indentation Experiments. Journal of Materials Research, 1992. 7(6): p. 1564-1583.

22. Li, X.D. and B. Bhushan, A review of nanoindentation continuous stiffness measurement technique and its applications. Materials Characterization, 2002. 48(1): p. 11-36.

23. Filleter, T., et al., Friction and Dissipation in Epitaxial Graphene Films. Physical Review Letters, 2009. 102(8).

a

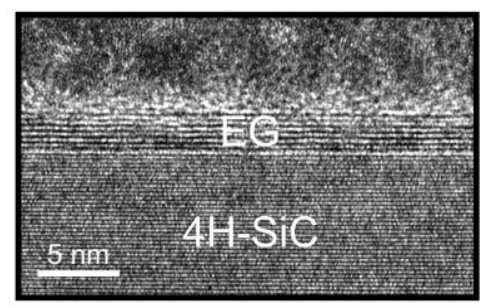

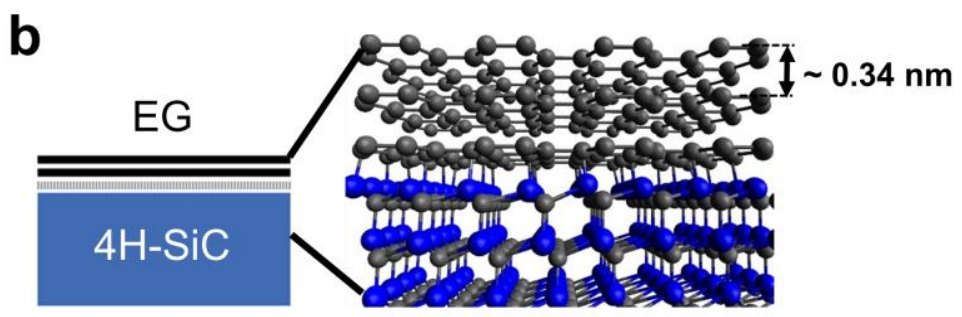

Fig. 1. (a) TEM image and (b) schematic illustration of few-layers epitaxial graphene grown on $\mathrm{SiC}$ (0001) substrate by $\mathrm{Si}$ sublimation at high temperatures. In the 3D lattice model, it is possible to distinguish the interfacial carbon buffer layer, which is directly bonded to the $\mathrm{SiC}$ substrate, and the graphene layers grown on top of it.

a

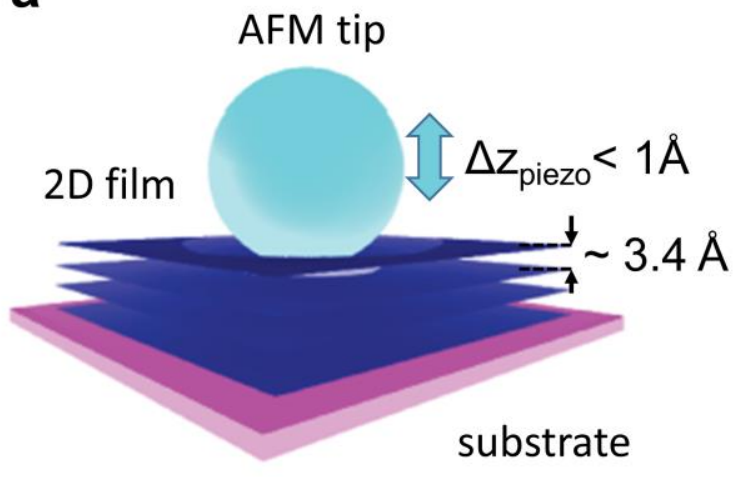

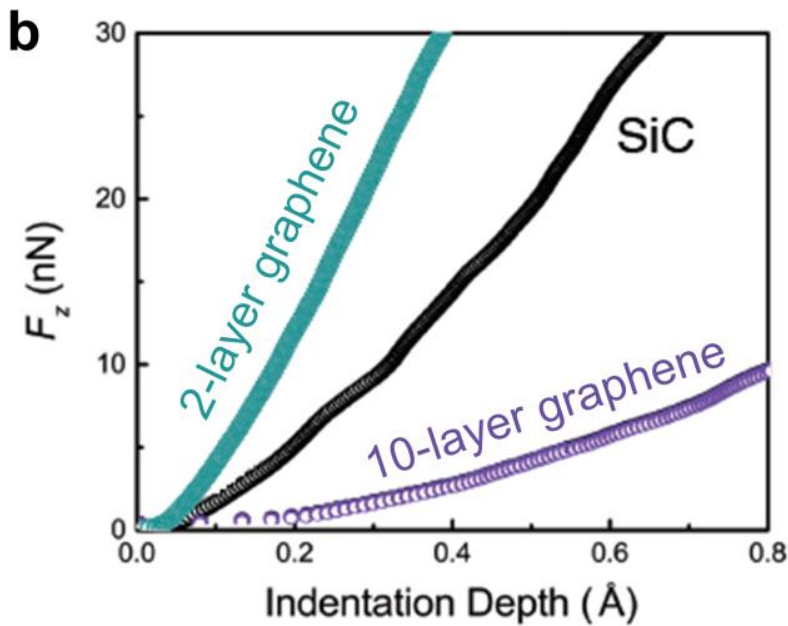

Fig. 2. (a) Schematic illustration of the contact model assumed in the Modulated Nanoindentation (MoNI) technique. The AFM tip can be represented as a sphere indenting locally the 2D film surface, assimilated, together with the substrate, to an elastic half-space. (b) Force versus indentation depths curves obtained from high-resolution Modulated Nanoindentation (MoNI) measurements, showing the mechanical responses of epitaxial graphene films of different thicknesses, compared to that of the SiC substrate. 

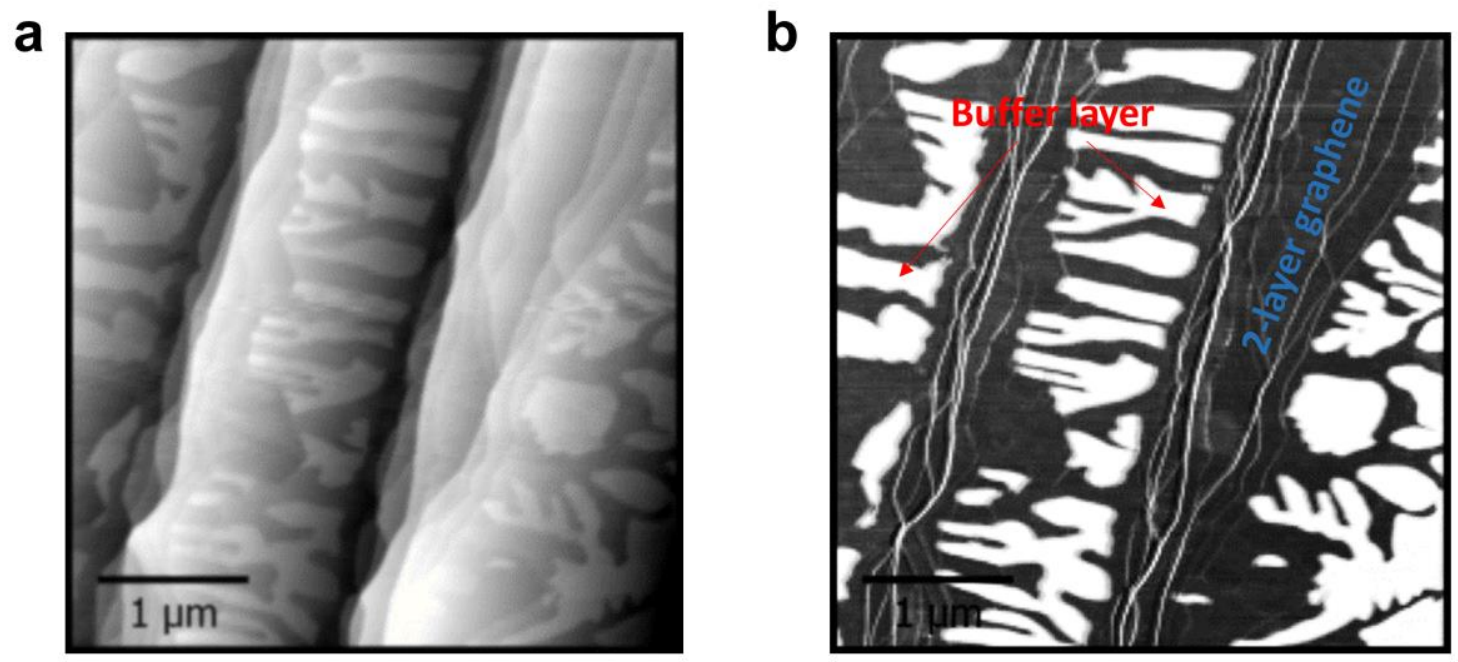

Fig. 3. (a) Contact-mode AFM topographic image (vertical scale: $10 \mathrm{~nm}$ ) of the 2-L epitaxial graphene sample studied in this work. Higher domains represent the buffer layer. (b) Corresponding friction force image (vertical scale: $50 \mathrm{nN}$ ). Thanks to the large difference in their friction response, from the friction map it is possible to clearly distinguish the exposed 2-L epitaxial graphene (low friction-dark contrast) form the buffer layer domains (high friction-brighter contrast). Friction force map has been acquired scanning the sample at $5 \mathrm{nN}$ normal load.
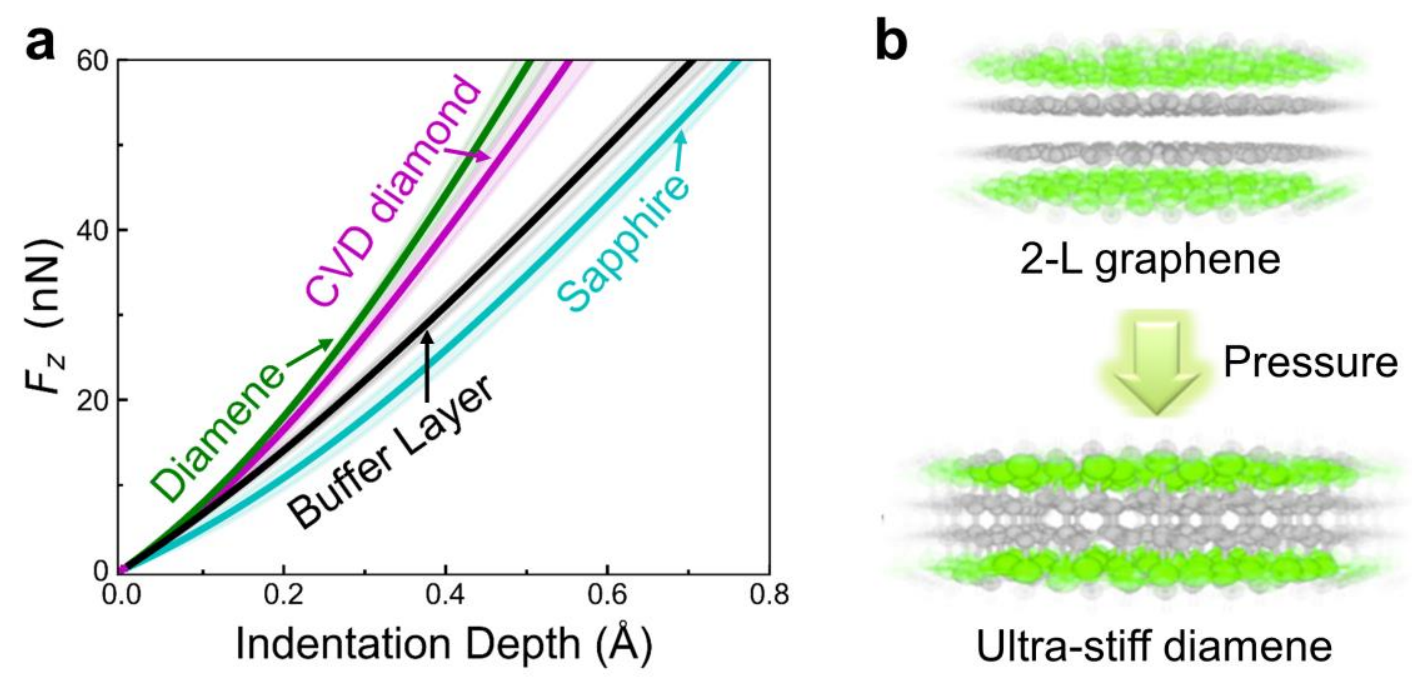

Ultra-stiff diamene

Fig. 4. (a) Force versus indentation depth curves obtained from MoNI experiments on 2-L epitaxial graphene on $\mathrm{SiC}(0001)$ and on different hard materials. The curves plotted are the result of an averaging process of data extracted from different positions on the surface of each sample. Shaded areas represent the respective standard deviations from the mean value. All the measurements are performed using the same diamond tip. The reported experiment clearly shows that the diamene/SiC structure (green line) exhibits a stiffness larger than that of CVD bulk diamond (magenta line). (b) Illustration of a 2-L epitaxial graphene film on $\mathrm{SiC}$ (represented by the bottom green atomic plane), undergoing the phase transition to a diamond-like structure (diamene) under the effect of compression exerted by the AFM tip (upper green atomic plane). 